Max-Planck-Institut für demografische Forschung

Max Planck Institute for Demographic Research

Konrad-Zuse-Strasse 1 - D-18057 Rostock - GERMANY

Tel +49 (0) 3812081 - 0; Fax +49 (0) 3812081 - 202;

http://www.demogr.mpg.de

MPIDR WORKING PAPER WP 2012-010

FEBRUARY 2012

\title{
Lifespan variation by occupational class: Compression or stagnation over time?
}

\author{
Alyson van Raalte (vanraalte@demogr.mpg.de) \\ Pekka Martikainen \\ Mikko Myrskylä (myrskyla@demogr.mpg.de)
}

(C) Copyright is held by the authors.

Working papers of the Max Planck Institute for Demographic Research receive only limited review. Views or opinions expressed in working papers are attributable to the authors and do not necessarily reflect those of the Institute. 


\title{
Lifespan variation by occupational class: Compression or stagnation over time?
}

\author{
Alyson van Raalte ${ }^{1}$, Pekka Martikainen ${ }^{2}$, Mikko Myrskylä ${ }^{1}$ \\ ${ }^{1}$ Max Planck Institute for Demographic Research, Rostock, Germany \\ ${ }^{2}$ Department of Sociology, University of Helsinki, Helsinki, Finland
}

\begin{abstract}
Adult lifespan variation in most western countries has stagnated since the 1960s, despite continued improvements in longevity. Cross-sectional analyses, however, find that in the 1990s higher socio-economic position was associated with lower lifespan variation. Trends in this association over time are unknown. We investigated trends in lifespan variation over four decades by occupational social class (manual, lower non-manual, upper non-manual) using Finnish register data (1971-2007). We performed age and cause-of-death decompositions of lifespan variation for each sex (a) by occupational class over time and (b) between occupational classes at a shared life expectancy. We found that although all occupational classes experienced increases in life expectancy, manual workers had stagnating lifespan variation over time while the higher occupational groups experienced mortality compression. These differences were caused by diverging trends in early adult mortality: all occupational classes experienced similar trends in lifespan variation at older ages, but variation in early adult mortality increased for all classes except the highest category. The high and stagnant lifespan variation of the manual class was mostly due to higher early adult mortality from external causes. These results suggest that mortality compression can be compatible with increases in life expectancy by tackling inequalities in early adult mortality.
\end{abstract}

Date of last revision: February 2012

Keywords: lifespan variation; life disparity; life expectancy; mortality compression; socioeconomic inequality; Finland; 


\section{Introduction}

Variation in lifespan is the 'final inequality' faced by individuals (Tuljapurkar 2010). Despite increases in life expectancy, adult lifespan variation, measured conditional upon surviving childhood, has mostly stagnated or shown only slight compression in western countries since the 1960s (Edwards 2011; Edwards and Tuljapurkar 2005; Engelman, Canudas-Romo and Agree 2010; Hill 1993; Kannisto 2000; Robine 2001; Smits and Monden 2009; Wilmoth and Horiuchi 1999). But analyses of national populations may hide important differences in subpopulations, for instance between socioeconomic groups. A cross-sectional analysis of lifespan variation by education in 10 European countries revealed a strong negative association between life expectancy and lifespan variation, both conditional upon survival to age 35, reproduced here as Figure 1 (van Raalte et al. 2011). Why life expectancy does not predict the level of adult lifespan variation at the country level over time, but does so cross-sectionally at the educational subgroup level, is unclear.

Figure 1 about here

Lifespan variation is important because it measures uncertainty in the timing of death. Reducing this uncertainty increases the value of both private and public investments in education and training, and can factor into life course decisions such retirement planning and the adoption of healthy behaviour. Moreover, the existence of substantial socioeconomic inequalities in lifespan variation may point to the failure of social protective policies (van Raalte et al. 2011). For this reason, trends in lifespan variation by socioeconomic status should be monitored alongside trends in life expectancy. 
Until now, however, studies on time trends in lifespan variation, including those that have examined causes of death, have only been performed at the national level. Papers that have focussed on socioeconomic inequalities in lifespan variation have been limited to cross-sectional analyses (van Raalte et al. 2011, Brown et al. in press) or to two points in time (Shkolnikov et al. 2003). We fill this void by being the first to examine how lifespan variation has changed since the 1970s for different socioeconomic groups. Using Finnish register data we test whether occupational social classes experienced similar trends in reducing lifespan variation and increasing life expectancy. We use occupational social class (hereafter occupational class) as our socioeconomic dimension because unlike education, its composition within the population has remained relatively stable. We compare the age and cause of death distribution of mortality for each occupational class over time and at similar levels of remaining life expectancy.

Our findings reveal stark differences by occupational class: increasing lifespan variation among manual workers and mortality compression among non-manual workers. These differences were due to diverging trends in early adult mortality. At shared life expectancies manual workers had higher lifespan variation at early adult ages owing to higher mortality from external causes, while differences in lifespan variation at older ages were negligible. These results suggest that continued declines in lifespan variation are possible alongside increases in life expectancy by tackling the high early adult mortality of the lower occupational classes. 


\section{Background and hypotheses}

The strength of the association in Figure 1 makes it tempting to interpret this relationship as a time trajectory_lower educated groups could simply lag behind the forerunner tertiary educated in reducing lifespan variation and increasing life expectancy. Or it could be that lifespan variation stagnated over time for all educational groups (i.e. absolute differences in lifespan variation between educational groups remained fixed), despite increases in life expectancy. Each finding would have its own implications. A compression scenario in lifespan variation by education would suggest that the highest educated acted as a vanguard group. This scenario could present itself if higher education or social status provided a pathway to adopting better health habits or to taking earlier advantage of medical breakthroughs, behaviours that eventually were transmitted down to lower educated individuals. A stagnating scenario would imply that individuals with lower education faced greater lifespan variation at all levels of life expectancy. This could be owing to persisting differences in environmental conditions, lifestyle and behaviour, or the psychosocial environment. That males and females were not on separate trajectories, as might have been imagined due to higher levels of early adult mortality among males (Oksuzyan et al. 2008), lends further weight to this latter hypothesis. Yet up until now, only a handful of studies have examined lifespan variation along a socioeconomic dimension (Brown et al. in press; Edwards and Tuljapurkar 2005; Shkolnikov, Andreev and Begun 2003; van Raalte et al. 2011). Although these studies found that groups with higher socioeconomic status enjoyed lower lifespan variation, they did not examine how this relationship evolved over time.

Lifespan variation increases or decreases depending on the balance between saving lives at early ages which compress the age-at-death distribution, and old ages which expand this distribution. Conceptually, lifespan variation should be split into 
these early and old age components which have different causes and theoretical implications (Vaupel, Zhang and van Raalte 2011). Examining lifespan variation at older ages is often done to determine whether mortality is being further compressed at these ages, which might signal a looming limit to the human lifespan in the absence of corresponding increases in the modal age at death, or alternatively, to establish whether the entire older age distribution of death is shifting to the right, known as the 'shifting mortality’ hypothesis (Bongaarts 2005; Canudas-Romo 2008; Kannisto 1996). A third theoretical model that is not mutually exclusive to the first two is the "postponement of selection' hypothesis. Under this framework, frail individuals, who in former times would not have survived the high disease environment, are increasingly being saved, postponing mortality selection to advanced ages (Bonneux, Barendregt and Van der Maas 1998; Engelman et al. 2010).

Empirically, although most countries appear to be experiencing continuing older age mortality compression alongside increases in the modal age at death (CanudasRomo 2008; Cheung, Robine and Caselli 2008; Kannisto 2000; Kannisto 2001; Ouellette and Bourbeau 2011; Thatcher et al. 2010) shifting mortality has been observed for both males and females in Japan since the 1990s (Canudas-Romo 2008; Cheung and Robine 2007; Ouellette and Bourbeau 2011; Thatcher et al. 2010), and early indications have been seen among females in France (Ouellette and Bourbeau 2011; Thatcher et al. 2010) and in Canada (Ouellette and Bourbeau 2011). No study that we know of has examined mortality compression at older ages in Finland.

Relatively fewer studies have interested themselves in differences between populations in early age mortality compression. This is surprising given that although only 38 percent of deaths from 1840 to the late 2000s of the Human Mortality Database were considered to be at infant and early adult ages, 84 percent of the gains in life 
expectancy came from averting infant and early adult mortality (Vaupel et al. 2011). Generally it has been shown through age decompositions that differences in the number of deaths over early adult ages (and infancy, when included) drive differences in lifespan variation between populations, with mortality differentials at older ages being relatively unimportant (Shkolnikov et al. 2003; Shkolnikov et al. 2011; van Raalte et al. 2011; Vaupel et al. 2011; Wilmoth and Horiuchi 1999). This is not always the case. Kibele (2011) demonstrated that throughout much of the 1970s and 1980s, the former East German states had lower lifespan variation (over ages 0-90) than West German states at similar levels of life expectancy, due not only to the East's lower young adult mortality, but also to its higher old age mortality.

\section{Data and Methods}

$\underline{\text { Data }}$

Our data consists of death and exposure counts by age (31-99+), sex, occupational class, and cause of death, covering the 1971-2007 period in Finland. The data is pulled together from two high-quality data sets, the first covering mortality over years 1988 to 2007 and the second over years 1971-2007. The later data set is based on individual level register data. It is an 11 percent sample of all persons residing in Finland in 19872007 obtained from Statistics Finland. The data was linked to death records by Statistics Finland by means of personal identification codes in the period 1988-2007. The data was complemented with an oversample of deaths so that 80 percent of all deaths during that period were included. To take account of this sampling design, weights were used to 'inflate' the person-years to match the $80 \%$ sample of deaths. Immigrants were dropped out since we did not have any baseline information on occupational class and emigrants were censored at emigration because their death records after emigration may 
be missed. This individual level data was then aggregated by sex, year, occupational class and age. In each year person days and deaths were allocated to one year age intervals between birthdays that are one month accurate.

The earlier data set was already tabulated by Statistics Finland. It comprises of four subsets that are based on information on census records for years 1970, 1975, 1980 and 1985. Census records were linked with cause of death records for periods 19711975, 1976-1980, 1981-1985 and 1986-1987 respectively. This data contains all persons and deaths during 1971-1987. Each five year period is closed to migration, as in the later data set. The overall death rates and social class differences in mortality obtained from these data are the same as those published elsewhere (Martikainen, Valkonen and Martelin 2001; Valkonen 1993).

In both data sets occupation-based socioeconomic status was measured at the time of each census updated in every $5^{\text {th }}$ year. . Four groups were distinguished: 1) upper non-manual, 2) lower non-manual, 3) manual worker and 4) other. The last group is comprised of farmers, entrepreneurs, students and those whose occupational status was unknown. The classification is retroactive i.e. for pensioners, unemployed persons and for those whose occupational status was unknown information was retrieved from earlier censuses. Those whose main activity was household work were classified according to the occupation of the head of the household. The proportion of the population in each occupational class is presented in Table 1.

Table 1 about here

We consider four major cause of death categories: circulatory diseases, neoplasms, external causes, and other causes. Circulatory diseases were included 
because of their obvious importance in mortality decline over the time frame examined. We considered neoplasms, because a high contribution from this cause might signal a strong role from smoking-related causes. Finally external causes are known to be socially patterned, and relatively high in Finland (Kunst et al. 1998). These causes were grouped from Statistics Finland's 54-class cause of death categories that has been harmonized over the different ICD versions.

Due to the small size of some of these occupational subgroups, data were aggregated over 5-year time intervals to increase the statistical power of the life table estimates (with 2001-2007 being a slightly longer interval) used in all analyses. This lowered the $95 \%$ confidence intervals around our remaining life expectancy estimate for the smallest subpopulation from around \pm 0.6 years to \pm 0.3 years ${ }^{1}$ and led to smoother age-at-death distributions. Lifetables were constructed by the methods described in the Human Mortality Database (Wilmoth et al. 2007) for each 5-year period, sex and occupational group. This included smoothing observed death rates from the age where the male death counts in the smallest occupational group (upper nonmanual) first fell below 100 deaths by fitting a Kannisto model (Thatcher, Kannisto and Vaupel 1998; Yi and Vaupel 2003). For each 5-year period, the same ages were smoothed for both sexes and all occupational classes. This also made it possible to extend the upper age limit from $99+$ to $110+$. By smoothing we compromised information on causes-of-death among the elderly. However, at these ages the interaction of multiple underlying causes of death make determining a single cause less reliable (Manton 1986; Minaker and Rowe 1985). Thus we do not carry out any cause of death comparisons over ages where mortality was smoothed, ranging from age 80 in the first period to age 94 in the last.

\footnotetext{
${ }^{1}$ Confidence intervals were estimated using Monte Carlo simulation methods, assuming a binomial distribution of death counts.
} 


\section{Measurement of lifespan variation}

Lifespan variation was measured using the life disparity conditional upon survival to age 31 index $\left(e_{31}^{\dagger}\right)$ (Vaupel and Canudas Romo 2003; Zhang and Vaupel 2009). We used life disparity because of its easy interpretation as the average years of life lost to death and because it can be divided into additive age components. This allowed us to separate the contributions of ages which compress mortality from ages which expand mortality. In recent years this index has become a popular option for measuring lifespan variation (Nusselder and Mackenbach 1996; Shkolnikov et al. 2011; Vaupel et al. 2011; Zhang and Vaupel 2009).

Life disparity is the sum of remaining life expectancy at each age, weighted by the number of deaths at that age:

$$
e_{31}^{\dagger}=\frac{\int_{31}^{\omega} f_{x} e_{x} d x}{\ell_{31}}
$$

where $f_{x}$ is the life table death density at age $x, e_{x}$ is remaining life expectancy, $\ell_{31}$ is survivorship at the starting age of the integral, here 31 years, and $\omega$ is the open-aged interval, which in our case is $110+{ }^{2}$

An alternative interpretation to the "average years of life lost to death" of $e_{31}^{\dagger}$ is the average remaining life expectancy at death conditional upon survival to age 31 . As deaths become compressed into a shorter age interval, on average individuals are dying with fewer years of remaining life expectancy, and $e_{31}^{\dagger}$ decreases. If everyone were to die at the same age it is easy to see from equation 1 that remaining life expectancy would be zero and hence $e_{31}^{\dagger}$ would equal zero as well. Life disparity $e_{x}^{\dagger}$ is also the

\footnotetext{
${ }^{2}$ These calculations were also performed for each period. For notational simplicity we dropped the subscript $t$ denoting time.
} 
numerator of Keyfitz' entropy measure $H$ (Goldman and Lord 1986; Vaupel 1986; Vaupel and Canudas Romo 2003), which Keyfitz himself noted was related to lifespan variation (Keyfitz 1977).

Compared to other indices of lifespan variation examined over adult ages, life disparity is sensitive to changes in mortality at later ages (Shkolnikov et al. 2011; van Raalte and Caswell 2012). Use of another measure would probably show wider differences in lifespan variation by occupational groups given that socioeconomic inequalities in lifespan variation tend to be driven by differences in early adult mortality to which life disparity is less sensitive (van Raalte et al. 2011). However the high correlations between measures of variability mean that broad conclusions would likely be the same regardless of the measure chosen (van Raalte and Caswell 2012; Vaupel et al. 2011; Wilmoth and Horiuchi 1999).

\section{$\underline{\text { Lifespan variation in early and old ages }}$}

Zhang and Vaupel (2009) showed that so long as $e_{x}^{\dagger}$ at a given age is less than remaining life expectancy at that age there exists one unique age, $a_{x}^{\dagger}$, that separates deaths which compress the age-at-death distribution from deaths which expand this distribution. ${ }^{3}$ Using our starting age of 31 , we then define early adult deaths as deaths occurring before age $a_{31}^{\dagger}$, while deaths occurring after this age are defined as old age mortality. This definition means that a death considered to be early adult will differ depending on the underlying mortality conditions. Intuitively this makes sense: when mortality is low deaths at for example age 70 are more unusual and 'early' than in high

\footnotetext{
3 This age is found by interpolation, setting the following function $k(a)$ equal to zero, $k(a)=e^{\dagger}(a)-e(a)(1-H(a))$ where $H(a)$ is the cumulative hazard function.
} 
mortality populations when deaths at such ages are common. ${ }^{4}$ Generally the threshold age sits just below the life expectancy (Vaupel et al. 2011). We divided $e_{31}^{\dagger}$ into early adult and old age components to see which component was driving the changes to lifespan variation,

$$
e_{31}^{\dagger}=\frac{\int_{31}^{a_{31}^{\dagger}} f_{x} e_{x} d x}{\ell_{31}}+\frac{\int_{a_{31}^{\dagger}}^{\omega} f_{x} e_{x} d x}{\ell_{\left(a_{31}^{\dagger}\right)}^{\dagger}}
$$

The close correspondence between lifespan variation and life expectancy has led some to argue that one should be examined within the context of the other (Smits and Monden 2009). For this reason, we not only compared lifespan variation over time, but also at similar levels of remaining life expectancy, regardless of the time period in which it happened.

\section{Decomposing a difference in lifespan variation by age and cause of death}

We decompose a difference in $e_{31}^{\dagger}$ by single year of age and cause of death by step-wise decomposition (Andreev, Shkolnikov and Begun 2002; Shkolnikov et al. 2011) using the VBA program developed by Shkolnikov and Andreev (2010). This general decomposition method can be applied to changes in any aggregate measure (including $e_{31}$ and $\left.e_{31}^{\dagger}\right)$ which depend only on the vectors of age-specific death rates, $\mathbf{M}$ and $\mathbf{M}^{\prime}$ from the two populations being compared. $\mathbf{M}$ itself is simply all cause mortality, or the

\footnotetext{
${ }^{4}$ However this definition also means that early adult deaths are different across social groups within a country. Thus some deaths that would be considered 'old age' among the lower social classes would be 'early adult' among the upper classes. Thus we consider 'early adult mortality' to be a technical definition and not to have any normative meaning.
} 
sum of age-specific death rates for all $n$ of the $i$ underlying causes (external, circulatory, neoplasms, other),

$$
\mathbf{M}=\left[\sum_{i=1}^{n} m_{0}^{i}, \sum_{i=1}^{n} m_{1}^{i}, \ldots, \sum_{i=1}^{n} m_{\omega}^{i}\right]
$$

We step-wise replaced each $m_{x}^{i}$ with that of the comparative population $m_{x}^{i}$, and recalculated $\mathbf{M}, e_{31}$ and $e_{31}^{\dagger}$ to determine the contribution, $\delta_{x}^{i}$, from each elementary age interval $[\mathrm{x}, \mathrm{x}+1)$ for cause $i^{5}$

We decomposed both differences over time for each occupational class, and differences between occupational classes at the same level of remaining life expectancy. This allowed us to determine whether the same age groups and causes of death that were driving differences over time were also driving differences between occupational classes at a given mortality level.

\section{Results}

\section{Comparisons over time}

Figure 2 about here

Figure 2 shows the life table age at death distributions by occupational class and sex 1971-1975 (the first period) and 2001-2007 (the last period). For all occupational categories and for both sexes, the distribution of deaths as a whole has moved to higher ages, suggesting that life expectancy has increased in all groups. However, for both men

\footnotetext{
${ }^{5}$ Small differences can arise when choosing to replace $m_{x}^{i}$ with $m_{x}^{i}$ ' or vice versa. Thus we performed the same procedure in reverse, and averaged the elementary contributions from each age and cause as suggested by Shkolnikov et al. (2011).
} 
and women in the manual class, a lingering left tail of the age-at-death distribution is seen despite the overall shift to the right. In contrast, visual inspection of the age-adeath distribution suggests upper non-manual workers experienced a marked compression in early adult deaths.

Figure 3 about here

Figure 3 shows the time trends in remaining life expectancy and life disparity at age 31 by occupational class for men and women. The results shown in Figure 3 confirm what visual inspection of the age-at-death distributions shown in Figure 2 suggested. Remaining life expectancy at age 31 increased for all occupational classes for both men (Panel A) and women (Panel B) in an almost parallel linear manner over the last 36 years (Figure 3-upper panels), with some increase of differentials as the pace of increase was somewhat faster in the higher occupational classes. Lifespan variation, however, showed strongly diverging trends by occupational class (Figure 3-lower panels). Among males (Panel C), the upper non-manual class experienced adult mortality compression during the entire period of study, while the lower non-manual and other class experienced mixtures of stagnation and compression. The manual class showed no reduction in lifespan variation over the period. Among females (Panel D), mortality compression was less pronounced. The manual class experienced stagnation and the other classes experienced a mixture of stagnation and compression.

Figure 4 about here 
Figure 4 shows trends in early adult and old age components of life disparity. The figure shows that the diverging trends in lifespan disparity were entirely driven by early adult mortality. Among both men (Panel A) and women (Panel B), the early adult component of lifespan variation slowly decreased for upper non-manual workers, increased sharply for manual workers and occupied varying intermediate positions for the other two subgroups. The old age component of lifespan variation, however, decreased over the time examined in all occupational classes for both men (Panel C) and women (Panel D). Despite similar trends, the level of old-age disparity for men varied by occupational class, being highest for lower classes.

Contributions of different causes of death to time trends

Figure 5 about here

Figure 5 shows what causes of death have contributed to the changing lifespan variation at different ages by decomposing the change in life disparity from 1971-75 to 2001-07 to age and cause-specific components for the manual and upper non-manual classes. Bars that are above the zero-line correspond to ages in which mortality change increased lifespan variation over the observation period; bars below the zero line correspond to ages in which mortality change decreased lifespan variation. Among male (Panel A) and female (Panel B) manual workers lifespan variation stagnated because the magnitude of compression in lifespan variation from reductions in early adult mortality roughly cancelled the expansion in lifespan variation from mortality improvement at older ages. In the upper non-manual classes (Panels C and D) the early adult mortality compression outpaced the expansive contribution from old age mortality reduction, 
leading to the overall compression. Overall, the temporal changes in lifespan variation overwhelmingly came from reductions in circulatory diseases. This effect was strongest in the non-manual classes both due to their later threshold age, which was above the age where most of the effect from reductions in circulatory diseases took place, and due to a greater intensity in circulatory mortality reduction at all ages. In the manual class, reductions in circulatory disease were also substantial, but balanced over early adult and older ages due to this group's younger threshold age. External cause mortality increased the lifespan variation of the manual class over ages 40 to 70 for both men and women but contributed relatively little to the overall change in lifespan variation in comparison to other causes of death.

\section{Comparisons at the same level of remaining life expectancy}

Figure 6 about here

At the same level of remaining life expectancy, the non-manual occupational classes always had lower levels of lifespan variation than the manual and other class. These differences widened over the observation period. Figure 6 shows trends in early adult and old age components of lifespan variation at different levels of remaining life expectancy at age 31 . The figure shows that the differences in the relationship between $e_{31}^{\dagger}$ and $e_{31}$ were driven by different levels of early adult mortality, which widened with increasing $e_{31}$. On average, the manual class had an early adult life disparity that was around one full year higher (males; Panel A) and half a year higher (females; Panel B) than the non-manual classes at similar remaining life expectancies. Among males, the old age mortality patterns were astonishingly similar across all occupational groups. 
Among females, the non-manual classes actually had higher older age life disparity at each level of remaining life expectancy.

\section{Contributions of different causes of death to social class differences}

Figure 7 about here

Next we performed age and cause-of-death decompositions of life disparity between the occupational classes when remaining life expectancy was similar, choosing an $e_{31}$ of roughly 43 years for males and 50.5 years females. ${ }^{6}$ We used the upper non-manual class as the reference. We excluded the group 'other' from the comparison since this group experienced large changes in its occupational composition over time.

The results are shown in Figure 7. Compared to the upper non-manual class of 1976-80, the manual class of 2001-07 had higher proportions of deaths at both the youngest and oldest ages, which led to $e_{31}^{\dagger}$ levels that were 0.9 years higher for men (Panel A) and 0.5 years higher for women (Panel B). The lower non-manual class (men Panel C; women Panel D) had similar overall $e_{31}^{\dagger}$ levels to the upper non-manual class, although with some differences in the age and cause pattern of mortality.

Differences in external cause mortality, especially below age 60, explained a substantial portion of the occupational class differences in life disparity. This held for males and females. For males above age 45, mortality from circulatory disease was lower for most ages in the lower non-manual and manual classes than it was in the upper non-manual class at the same level of life expectancy. This points to strong

\footnotetext{
${ }^{6}$ Males: upper non-manual $(1976-80) e_{31}=43.1$ years, $e_{31}^{\dagger}=10.0$; lower non-manual $(1986-90) e_{31}=43.4$, $e_{31}^{\dagger}=10.2 ;$ manual workers (2001-07), $e_{31}=43.6$ years, $e_{31}^{\dagger}=10.9$.

Females: upper non-manual (1981-86) $e_{31}=50.2, e_{31}^{\dagger}=8.7$; lower non-manual $(1986-1990) e_{31}=50.5, e_{31}^{\dagger}=$ 8.5 ; manual workers (2001-2007) $e_{31}=50.6$ years $e_{31}^{\dagger}=9.2$.
} 
advances against circulatory conditions that took place between when the upper nonmanual group had an $e_{31}$ of 43 (1976-80) and when this average mortality level was reached by the lower non-manual (1986-90) and manual (2001-07) occupational classes. Overall, however, mortality from circulatory diseases had little effect on the life disparity differentials between subgroups, as the gains to the lifespan were spread over ages that both compressed and expanded the age-at-death distribution. The pattern was less clear among females, as circulatory disease mortality was higher for the lower occupational groups in the youngest ages (31-60) and lower for these groups at older ages. Mortality from other causes including neoplasms was relatively unimportant in explaining the differences in life disparity between occupational classes at similar life expectancy levels.

The role of external cause mortality

Figure 8 about here

The results from the last section point to a strong role played by external cause mortality in explaining between-group differences in lifespan variation. To quantify the importance of external cause mortality we calculated external cause-deleted life tables and compared the resulting $e_{31}^{\dagger}$ of the occupational groups over time and at the same level of remaining life expectancy. ${ }^{7}$ The results are shown in Figure 8. For men (Panels A and C), eliminating external cause mortality reduced adult lifespan variation more for the manual class than it did for the non-manual classes and resulted in all occupational

\footnotetext{
${ }^{7}$ Since we can assume a reasonable degree of independence between external cause mortality and other causes of death, cause-elimination can be a particularly effective tool to gauge the impact of external cause mortality on life expectancy and life disparity.
} 
classes being on a similar lifespan variation $\sim$ remaining life expectancy trajectory. For women (Panels B and D) eliminating external cause mortality had a similar narrowing effect on differences in life disparity, though the initial differences were lower.

Thus in the absence of external cause mortality, the lower occupational classes would have experienced similar levels of lifespan variation to the upper occupational classes at a shared level of life expectancy. Moreover, since all occupational classes were on a similar upward life expectancy trajectory (Figure 3), the trajectory of lifespan variation would also have similarly trended downward for all groups, only lagged for the lower occupational groups.

\section{Discussion}

\section{$\underline{\text { Summary of the main findings }}$}

The objective of this study was to determine whether - in a period of increasing life expectancy - lifespan variation by occupational class stagnated or showed compression over time. In fact we found different patterns across these occupational groupings. The lower occupational classes experienced stagnating lifespan variation while the higher occupational classes experienced mortality compression, owing to diverging trends in early adult mortality. Although circulatory diseases were driving trends in lifespan variation over time for all occupational classes, differences in external cause mortality explained much of the differences between occupational classes at the same level of remaining life expectancy.

\section{Early adult and older age components of lifespan variation}

An advantage to our study was the ability to disentangle the changes in lifespan variation by an early age component leading to mortality compression and an old age 
mortality component leading to mortality expansion. Of the two, trends in lifespan variation by occupational class in Finland were driven by the early adult component, which is in line with most macro level studies (Vaupel et al. 2011; Wilmoth and Horiuchi 1999). Thus, the lower occupational groups experienced increasing lifespan variation due to stagnation in early adult mortality, while the higher occupational groups experienced comparatively greater reductions in mortality over ages which compress the age at death distribution.

At any given time, the non-manual occupational classes had lower lifespan variation at old ages than the manual classes. However, the downward time trend was similar for all groups and when measured at similar life expectancy levels, the nonmanual classes did not have lower lifespan variation at these ages. The only other study to examine old age mortality compression along a socioeconomic dimension was Brown et al's (in press) study of the United States. They found a positive association between education and mortality compression. Unfortunately they were unable to examine trends in this relationship, to determine whether the more compressed old age mortality of the higher educated groups was associated with their higher overall life expectancy, as we have found here for Finland.

By examining lifespan variation above the threshold age rather than the mode as a measure of old age compression, we found no conclusive evidence of shifting mortality among males or among the middle to lower female occupational classes. The female upper non-manual occupational class however experienced compression in old age lifespan variation during every period except the last, which showed a slight uptake. Whether this is the beginning of a new era of shifting mortality remains to be seen. 
Comparisons to previous studies of socioeconomic differences in lifespan variation

Longer-term trends in lifespan variation along a socioeconomic dimension are unknown for other countries and only a few studies have examined this dimension in the crosssection, most of which used older datasets. Shkolnikov et al. (2003) found higher lifespan variation among lower educated Russians (ages 20 to 65) with widening differences by educational group between the two periods examined, 1979 and 1989. Although the age groups are not directly comparable to our own, in Russia all subgroups experienced some early adult mortality compression during this time, which the authors attributed to a decrease in alcohol-related mortality resulting from Gorbachev's anti-alcohol campaign. In Finland, trends were opposite during this period - the manual occupational class experienced increasing early adult lifespan variation throughout the 1980s while the non-manual classes experienced early adult mortality compression.

Socioeconomic inequalities in lifespan variation have also been shown in the United States. Edwards and Tuljapurkar (2005) found a 10 to 15 percent difference in lifespan variation (ages 10+) by either income or education using 1980 data from the National Mortality Longitudinal Survey. A more recent examination of the US was conducted by Brown et al. (in press) using data from the Health and Retirement Study (aggregated over 1992-2006) for the population aged 50+. Unfortunately they only calculated lifespan variation at ages above the mode, hampering direct comparison to our study. Visual comparisons of their depicted death densities by levels of education, however, reveal socioeconomic differences in lifespan variation similar in magnitude to those depicted for Finland in Figure 4. Finally van Raalte et al. (2011) compared lifespan variation (ages 35+) in 10 European countries over the 1990s. Finland had larger socioeconomic inequalities in lifespan variation than other northern and western 
European countries (apart from males in Switzerland), but smaller inequalities than in Eastern Europe. This was due in part to the large external mortality component in Finland found among younger adults.

Although little is known about socioeconomic trends in lifespan variation, there is a vast literature about socioeconomic inequalities in mortality, most of which cover adult mortality over ages that we have defined here as early adult. Finland had moderately high levels of educational inequality in all-cause mortality in the 1990s (Mackenbach et al. 2008), comparable to levels found in the United States (Mackenbach et al. 1999). In part these trends could be attributed to greater widening of occupational inequalities in mortality over early adult ages (30-74) in Finland during the 1980s. Faster declines in mortality among higher occupational groups, especially from cardiovascular diseases, drove the diverging trends (Mackenbach et al. 2003). Given that this is the age range driving changes in lifespan variation, we would also expect a greater widening of socioeconomic differences in lifespan variation to have taken place in the 1980s in Finland than in the other northern European countries. In addition to cardiovascular disease, a higher contribution from external causes of death also played a role in the moderately high socioeconomic inequalities in Finland (Kunst et al. 1998). Binge drinking was particularly problematic (Simpura, Paakkanen and Mustonen 1995), and was associated with $47 \%$ (males) and 17\% (females) of all external cause mortality cases (Mäkelä, Valkonen and Martelin 1997). Although the high external cause mortality in Finland among manual classes may in large part be related to its binge drinking culture (Kauhanen et al. 1997), a socioeconomic gradient to external cause mortality was found across Europe (Kunst et al. 1998) but was less pronounced among females (Mackenbach et al. 1999). In the United States, Edwards and Tuljapurkar (2005) found that external cause mortality accounted for a little less than one-tenth of 
the total standard deviation in lifespan conditional upon survival to age 10 in the United States (both sexes combined) — results very similar to our own. Unfortunately they did not examine external cause mortality by socioeconomic group. From these studies we expect that external cause mortality likely plays a larger role in Finland in explaining socioeconomic differences in lifespan variation than in other western European countries, especially at similar levels of life expectancy, but that its contribution to lifespan variation may be similar or smaller to that in the United States and in Eastern Europe.

The contribution of causes of death to socioeconomic differences in lifespan variation

We are not the first to notice that between-population differences in lifespan variation in the cross-section arise from different causes than within-population differences over time, although to our knowledge we are the first to examine this issue along a socioeconomic dimension. Reductions in chronic conditions such as circulatory diseases and certain cancers were found to have played a greater role in explaining withincountry changes in lifespan variation over time than they did in cross-country or crosssex comparisons at any given time (Shkolnikov et al. 2011; Zureick 2010).

The finding that the old age mortality component of lifespan variation is similar across occupational classes at the same level of life expectancy is good news. Although at a higher risk from early adult mortality, especially from external causes, once the lower classes survive to a certain age they experience similar mortality processes to the upper classes - with a lag. This result is consistent with speculation that much of the particularly rapid widening of socioeconomic inequalities in Finland over the 1980s owed to the earlier uptake of coronary surgeries and new methods of treatment for 
cardiovascular diseases by the non-manual classes (Martikainen et al. 2001; Valkonen et al. 2000).

\section{$\underline{\text { Methodological considerations }}$}

We measured adult lifespan variation by the life disparity measure $e_{31}^{\dagger}$. We studied the robustness of the results to alternative indices of lifespan variation by replicating all analyses with the Gini coefficient and the standard deviation (results not shown). None of the conclusions changed.

We used age 31 as the starting age of the age-at-death distribution. The starting age influences lifespan variation measures, in particular if infant and child mortality are included (Engelman et al. 2010; Nusselder and Mackenbach 1996; Robine 2001; van Raalte and Caswell 2012). We used a starting age that was old enough to contain useful information on occupational class, but young enough to capture the dynamics of early adult mortality. Although we would not expect large differences in measuring lifespan variability with a starting age in the 25 to 40 age range (i.e. once the occupational status is established but before the onset of most adult mortality), an analysis that examined mortality after some later adult age might yield substantively different results.

We used occupational class as our measure of socioeconomic status because compared to education there was less compositional change over the 37 years of observation. In particular, the reduction of the manual class from 49 to 45 percent of the population was minimal. Thus it is unlikely that the stagnating or even increased lifespan variation over time of manual workers was due to this class becoming composed of an increasingly selected group of individuals.

The period we studied covered several revisions to the ICD coding of disease, which can introduce breaks in the time series for certain diseases which might 
artificially inflate the change in contribution of certain diseases to lifespan variation over time. Any bias this might introduce should be minimal, since changes are likely to be within each broad category of disease that we created rather than between categories. Likewise, we did not notice any jump in trends in moving between ICD codes or between the two data sets provided by Statistics Finland. More generally, given the high quality of the Finnish dataset we expect our results and conclusions to be robust.

Our study has many advantages over other similar studies that have examined lifespan variation along a socioeconomic dimension. Unlike American studies which have had to rely on survey data (Brown et al. in press; Edwards and Tuljapurkar 2005), this study made use of the Finnish population registry which included the institutionalized population and could give a more accurate profile of mortality compression or expansion among the elderly. To date the European studies have been census-based and either examined trends between two censuses (Shkolnikov et al. 2003) or aggregated data over an intercensal period (van Raalte et al. 2011). We were fortunate to have yearly data by single year of age which allowed us to more carefully disentangle differences in mortality compression and expansion. Finally this study contained the longest time frame under examination and was the first to examine time trends in lifespan variation along a socioeconomic dimension in a country with continually improving life expectancy.

\section{$\underline{\text { Conclusion }}$}

Examining the variation in lifespans complements studies that monitor trends in life expectancies. That lower socioeconomic classes experience higher lifespan variation at all levels of life expectancy is a further reminder of the hardships they face. The results from this study suggest that policies to reduce lifespan variation should address the high 
mortality from accidents and violence of the lower occupational classes. This should be coupled with continued efforts to reduce disparities in access to new medical treatments and campaigns to discourage deleterious behaviour.

Finally this study showed that continued mortality compression can be compatible with increases in life expectancy, as the example of the upper non-manual class showed. To continue to do so requires tackling the high early adult mortality of the lower socioeconomic groups, especially the high mortality from external causes.

\section{References}

Andreev, E., V. Shkolnikov, and A.Z. Begun. 2002. "Algorithm for decomposition of differences between aggregate demographic measures and its application to life expectancies, healthy life expectancies, parity-progression ratios and total fertility rates." Demographic Research 7(14):499-522.

Bongaarts, J. 2005. "Long-range trends in adult mortality: models and projection methods." Demography 42(1):23-49.

Bonneux, L., J.J. Barendregt, and P.J. Van der Maas. 1998. "The expiry date of man: a synthesis of evolutionary biology and public health." Journal of epidemiology and community health 52(10):619.

Brown, D.C., M. Hayward, J.K. Montez, R.A. Hummer, C.-T. Chiu, and M.M. Hidajat. in press. "The Significance of Education for Mortality Compression in the United States." Demography.

Canudas-Romo, V. 2008. "The modal age at death and the shifting mortality hypothesis." Demographic Research 19(30):1179-1204.

Cheung, S.L. and J.M. Robine. 2007. "Increase in common longevity and the compression of mortality: the case of Japan." Population Studies 61(1):85-97.

Cheung, S.L.K., J.M. Robine, and G. Caselli. 2008. "The use of cohort and period data to explore changes in adult longevity in low mortality countries." Genus 65(12):104-129.

Edwards, R.D. 2011. "Changes in world inequality in length of life: 1970-2000." Population and Development Review 37(3):499-528.

Edwards, R.D. and S. Tuljapurkar. 2005. "Inequality in life spans and a new perspective on mortality convergence across industrialized countries." Population and Development Review 31(4):645-674.

Engelman, M., V. Canudas-Romo, and E.M. Agree. 2010. "The implications of increased survivorship for mortality variation in aging populations." Population and Development Review 36(3):511-539.

Goldman, N. and G. Lord. 1986. "A new look at entropy and the life table." Demography 23(2):275-282.

Hill, G. 1993. "The entropy of the survival curve: an alternative measure." Canadian Studies of Population 20(1):43-57. 
Kannisto, V. 1996. The advancing frontier of survival: Life tables for old age.

Monographs on Population Aging 3, Odense University Press.

—. 2000. "Measuring the compression of mortality." Demographic Research 3(6).

—. 2001. "Mode and dispersion of the length of life." Population: An English Selection 13(1):159-171.

Kauhanen, J., G.A. Kaplan, D.E. Goldberg, and J.T. Salonen. 1997. "Beer binging and mortality: results from the kuopio ischaemic heart disease risk factor study, a prospective population based study "BMJ 315:846.

Keyfitz, N. 1977. Applied mathematical demography. New York: Wiley.

Kibele, E. 2011. Regional mortality differentials in Germany: Spatial patterns, temporal trends, contextual and individual-level determinants. Rostock: $\mathrm{PhD}$ dissertation, University of Rostock.

Kunst, A.E., F. Groenhof, J.P. Mackenbach, and D.A. Leon. 1998. "Occupational class and cause specific mortality in middle aged men in 11 European countries: comparison of population based studies." BMJ 316(7145):1636-1642.

Mackenbach, J.P., V. Bos, O. Andersen, M. Cardano, G. Costa, S. Harding, A. Reid, O. Hemstrom, T. Valkonen, and A.E. Kunst. 2003. "Widening socioeconomic inequalities in mortality in six Western European countries." International Journal of Epidemiology 32(5):830-837.

Mackenbach, J.P., A.E. Kunst, F. Groenhof, J.K. Borgan, G. Costa, F. Faggiano, P. Jozan, M. Leinsalu, P. Martikainen, J. Rychtarikova, and T. Valkonen. 1999. "Socioeconomic inequalities in mortality among women and among men: an international study." American Journal of Public Health 89(12):1800-1806.

Mackenbach, J.P., I. Stirbu, A.-J.R. Roskam, M.M. Schaap, G. Menvielle, M. Leinsalu, A.E. Kunst, and H. the European Union Working Group on Socioeconomic Inequalities in. 2008. "Socioeconomic inequalities in health in 22 european countries." New England Journal of Medicine 358(23):2468-2481.

Mäkelä, P., T. Valkonen, and T. Martelin. 1997. "Contribution of deaths related to alcohol use to socioeconomic variation in mortality: register based follow up study." BMJ 315(7102):211-216.

Manton, K.G. 1986. "Cause specific mortality patterns among the oldest old: multiple cause of death trends 1968 to 1980 " Journal of Gerontology 41(2):282-289.

Martikainen, P., T. Valkonen, and T. Martelin. 2001. "Change in male and female life expectancy by social class: decomposition by age and cause of death in Finland 1971-95." Journal of Epidemiology and Community Health 55(7):494-499.

Minaker, K.L. and J. Rowe. 1985. "Health and disease among the oldest old: a clinical perspective." The Milbank Memorial Fund Quarterly. Health and Society 63(2):321-349.

Nusselder, W.J. and J.P. Mackenbach. 1996. "Rectangularization of the survival curve in The Netherlands, 1950-1992." Gerontologist 36(6):773-782.

Oksuzyan, A., K. Juel, J.W. Vaupel, and K. Christensen. 2008. "Men: good health and high mortality. Sex differences in health and aging." Aging clinical and experimental research 20(2):91.

Ouellette, N. and R. Bourbeau. 2011. "Changes in the age-at-death distribution in four low mortality countries: A nonparametric approach." Demographic Research 25(19):595-628.

Robine, J.-M. 2001. "Redefining the stages of the epidemiological transition by a study of the dispersion of life spans: the case of France." Population: An English Selection 13(1):173-193. 
Shkolnikov, V., E. Andreev, and A.Z. Begun. 2003. "Gini coefficient as a life table function. Computation from discrete data, decomposition of differences and empirical examples." Demographic Research 8:305-358.

Shkolnikov, V.M. and E.M. Andreev. 2010. "Age-decomposition of a difference between two populations for any life-table quantity in Excel." Rostock, Germany. Max Planck Institute for Demographic Research. TR 2010-002 downloaded 28/06/2010.

Shkolnikov, V.M., E.M. Andreev, Z. Zhang, J. Oeppen, and J.W. Vaupel. 2011. "Losses of expected lifetime in the United States and other developed countries: methods and empirical analyses." Demography 48(1):211-239.

Simpura, J., P. Paakkanen, and H. Mustonen. 1995. "New beverages, new drinking contexts - signs of modernization in Finnish drinking habits from 1984 to 1992, compared with trends in European community." Addiction 90(5):673-683.

Smits, J.and C. Monden. 2009. "Length of life inequality around the globe." Social Science \& Medicine 68(6):1114-1123.

Thatcher, A.R., S.L.K. Cheung, S. Horiuchi, and J.-M. Robine. 2010. "The compression of deaths above the mode." Demographic Research 22(17):505-538.

Thatcher, R.A., V. Kannisto, and J.W. Vaupel. 1998. The force of mortality at ages 80 to 120. Odense: Odense University Press.

Tuljapurkar, S. 2010. "The final inequality: variance in age at death." in Demography and the Economy, edited by J.B. Shoven: NBER, University of Chicago Press.

Valkonen, T. 1993. "Problems in the measurement and international comparison of socio-economic differences in mortality." Social Science \& Medicine 36(4):409418.

Valkonen, T., P. Martikainen, M. Jalovaara, S. Koskinen, T. Martelin, and P. Mäkelä. 2000. "Changes in socioeconomic inequalities in mortality during an economic boom and recession among middle-aged men and women in Finland." The European Journal of Public Health 10(4):274-280.

van Raalte, A.A., A.E. Kunst, P. Deboosere, M. Leinsalu, O. Lundberg, P. Martikainen, B.H. Strand, B. Artnik, B. Wojtyniak, and J.P. Mackenbach. 2011. "More variation in lifespan in lower educated groups: evidence from 10 European countries." International Journal of Epidemiology 40(6):1703-1714.

van Raalte, A.A. and H. Caswell. 2012. "Perturbation analysis of measures of lifespan variability." MPIDR Working Paper WP-2012-004.

Vaupel, J.W. 1986. "How change in age-specific mortality affects life expectancy." Population Studies 40(1):147-157.

Vaupel, J.W. and V. Canudas Romo. 2003. "Decomposing change in life expectancy: a bouquet of formulas in honor of Nathan Keyfitz's 90th birthday." Demography 40(2):201-216.

Vaupel, J.W., Z. Zhang, and A.A. van Raalte. 2011. "Life expectancy and disparity." BMJ Open 1:e000128. doi:10.1136/bmjopen-2011-000128

Wilmoth, J.R., K. Andreev, D. Jdanov, and D. Glei. 2007. "Methods protocol for the human mortality database (version 5)."

Wilmoth, J.R. and S. Horiuchi. 1999. "Rectangularization revisited: variability of age at death within human populations." Demography 36(4):475-495.

Yi, Z. and J.W. Vaupel. 2003. "Oldest-old mortality in China." Demographic Research $8(7): 215-244$.

Zhang, Z. and J.W. Vaupel. 2009. "The age separating early deaths from late deaths." Demographic Research 20:721-730. 
Zureick, S. 2010. Certainty in timing of death: a new analysis of shifting mortality and life span disparity. Berkeley: $\mathrm{PhD}$ dissertation, University of California at Berkeley. 


\section{TABLES AND FIGURES}

\begin{tabular}{lccccccc}
\hline & $1971-75$ & $1976-80$ & $1981-85$ & $1986-1990$ & $1991-1995$ & $1996-2000$ & $2001-2007$ \\
\hline Manual workers & 48.9 & 48.1 & 50.1 & 48.3 & 46.8 & 45.8 & 45.1 \\
Lower non-manual & 14.1 & 15.9 & 15.8 & 16.7 & 17.1 & 17.4 & 18.6 \\
Upper non-manual & 8.4 & 11.2 & 12.6 & 14.0 & 15.1 & 15.7 & 16.9 \\
Other workers & 28.6 & 24.8 & 21.4 & 20.9 & 21.0 & 21.1 & 19.4 \\
\hline
\end{tabular}

Table 1: The population proportion by occupational class for each period under observation. Data are from Statistics Finland. 


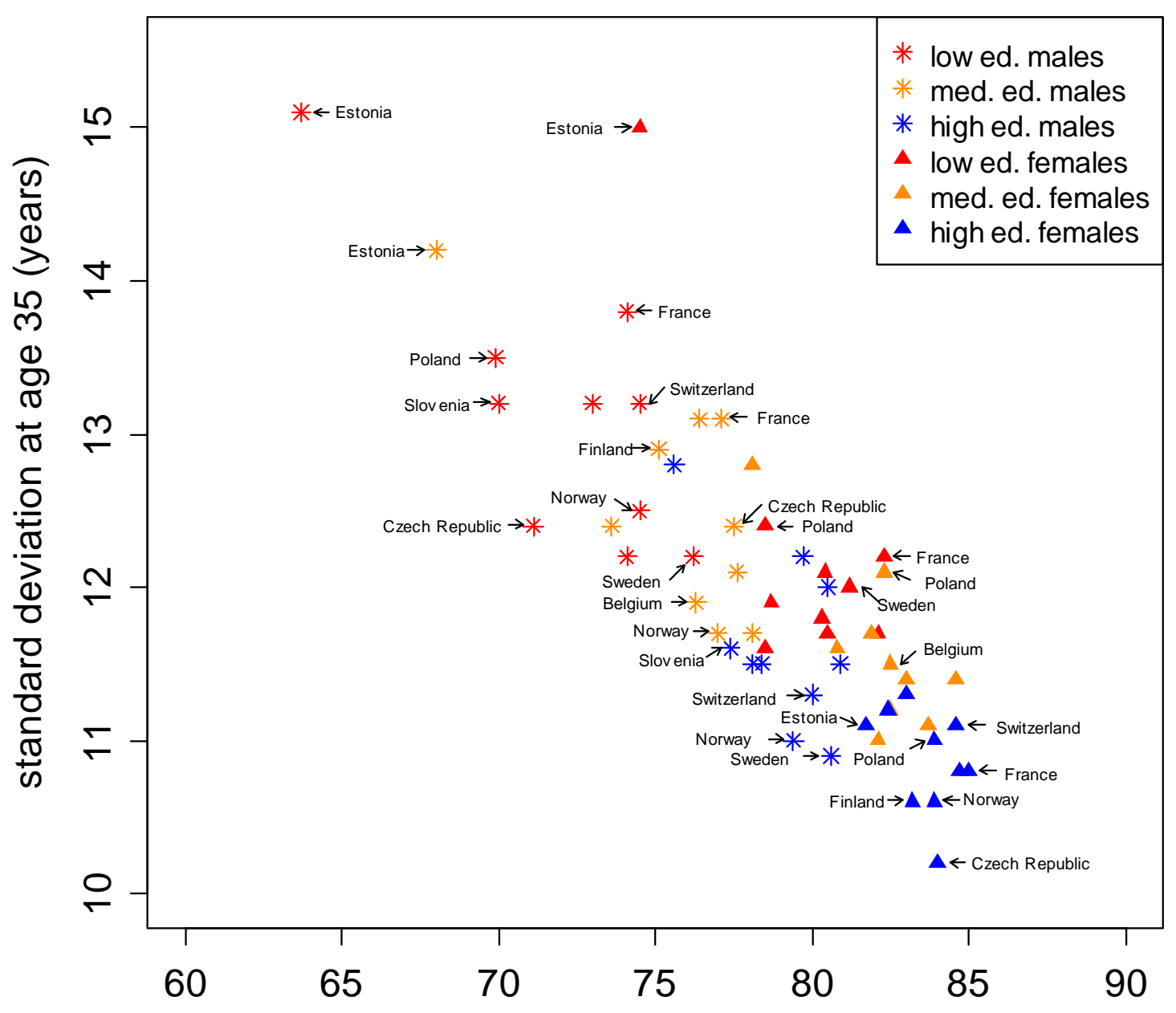

average age at death conditional upon survival to age 35

Figure 1: Cross-sectional relationship between lifespan variation and the average lifespan, both conditional upon survival to age 35, by sex and level of education. The data covers a period ranging from 1990-2003. The figure is reproduced from van Raalte et al. (2011). 

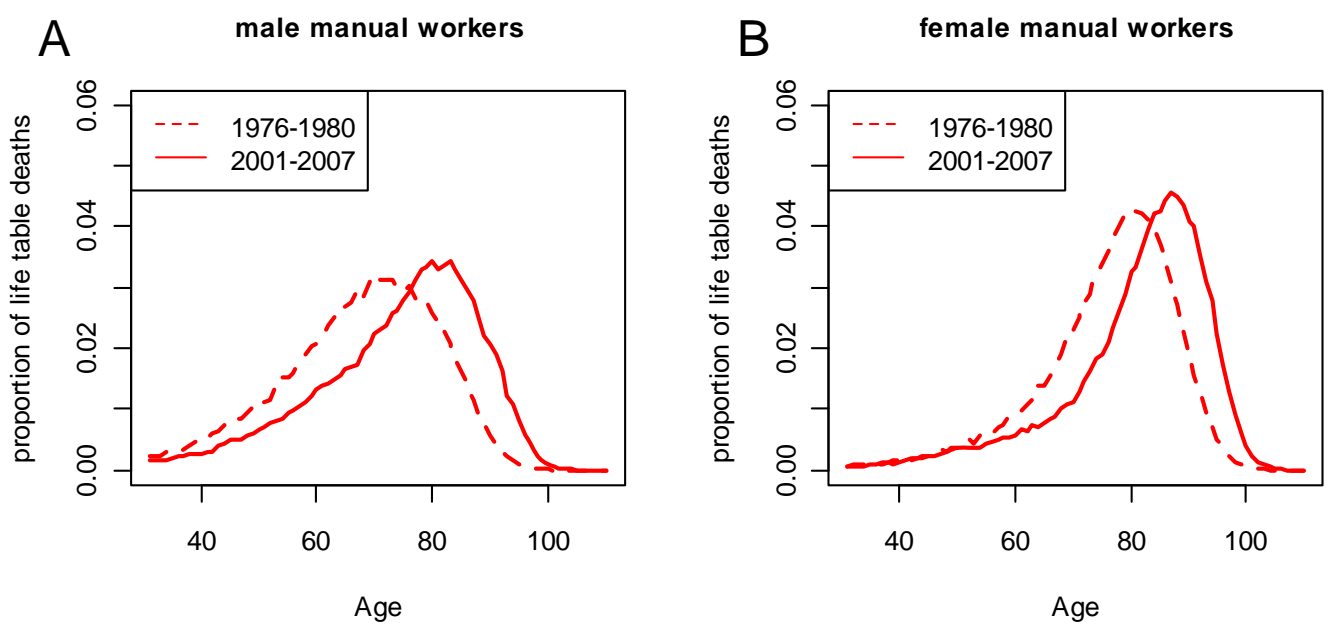

C

D female lower non-manual workers
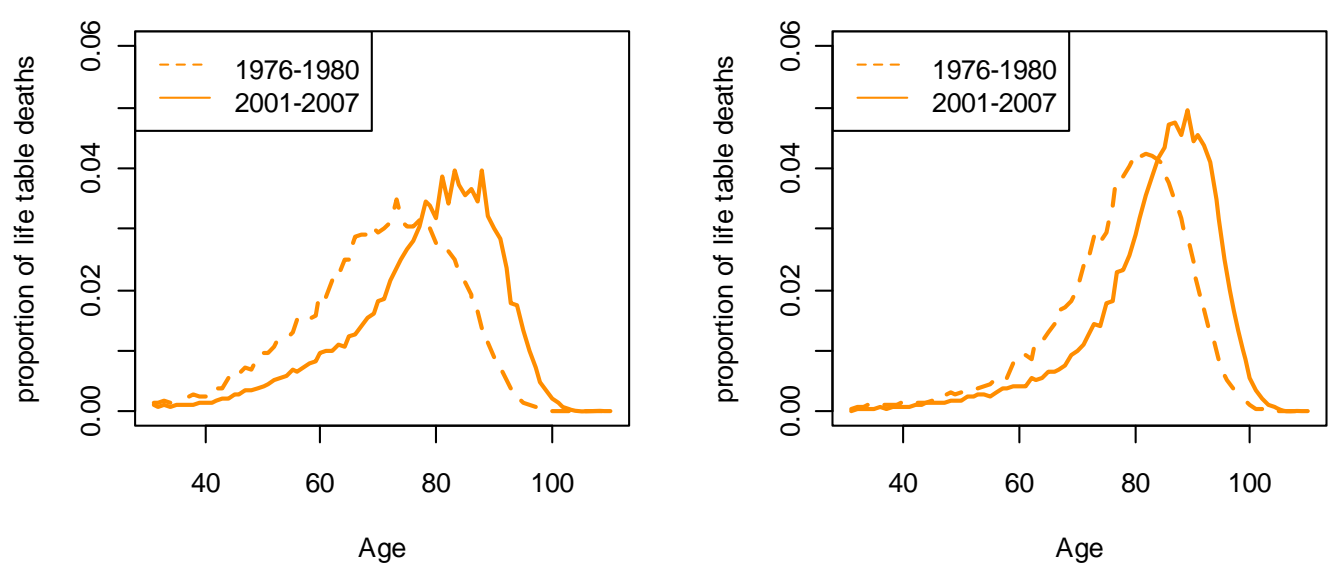

$E$

F female upper non-manual workers
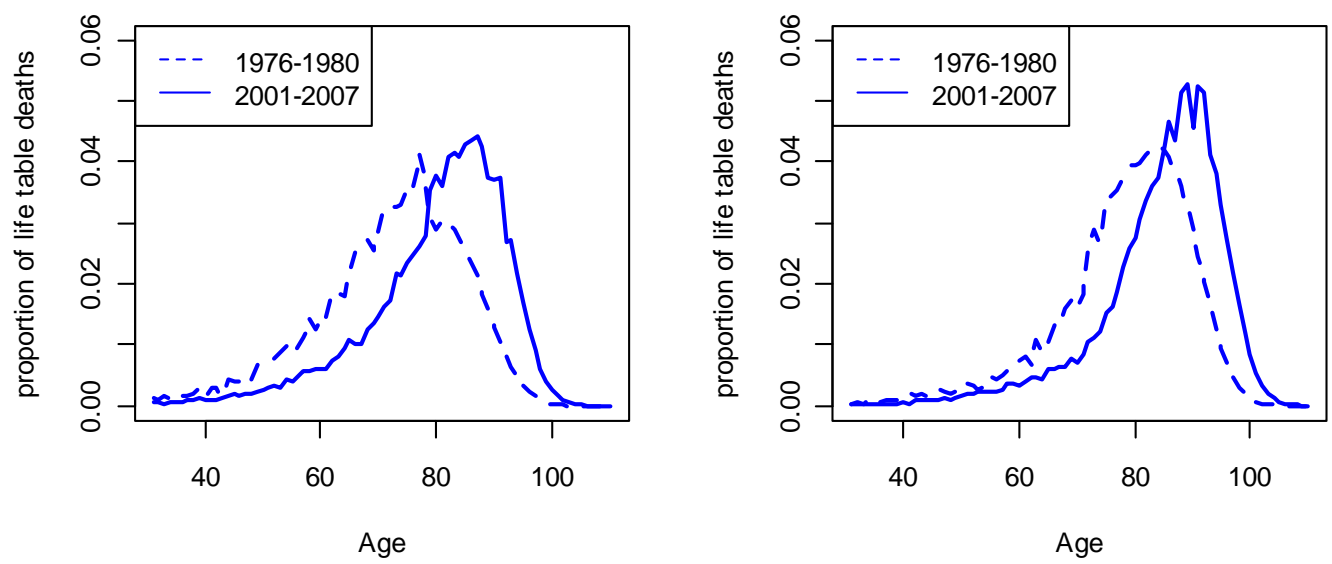

Figure 2: The change in life table age-at-death distributions conditional upon survival to age 31 for the different occupational classes, Finland. 

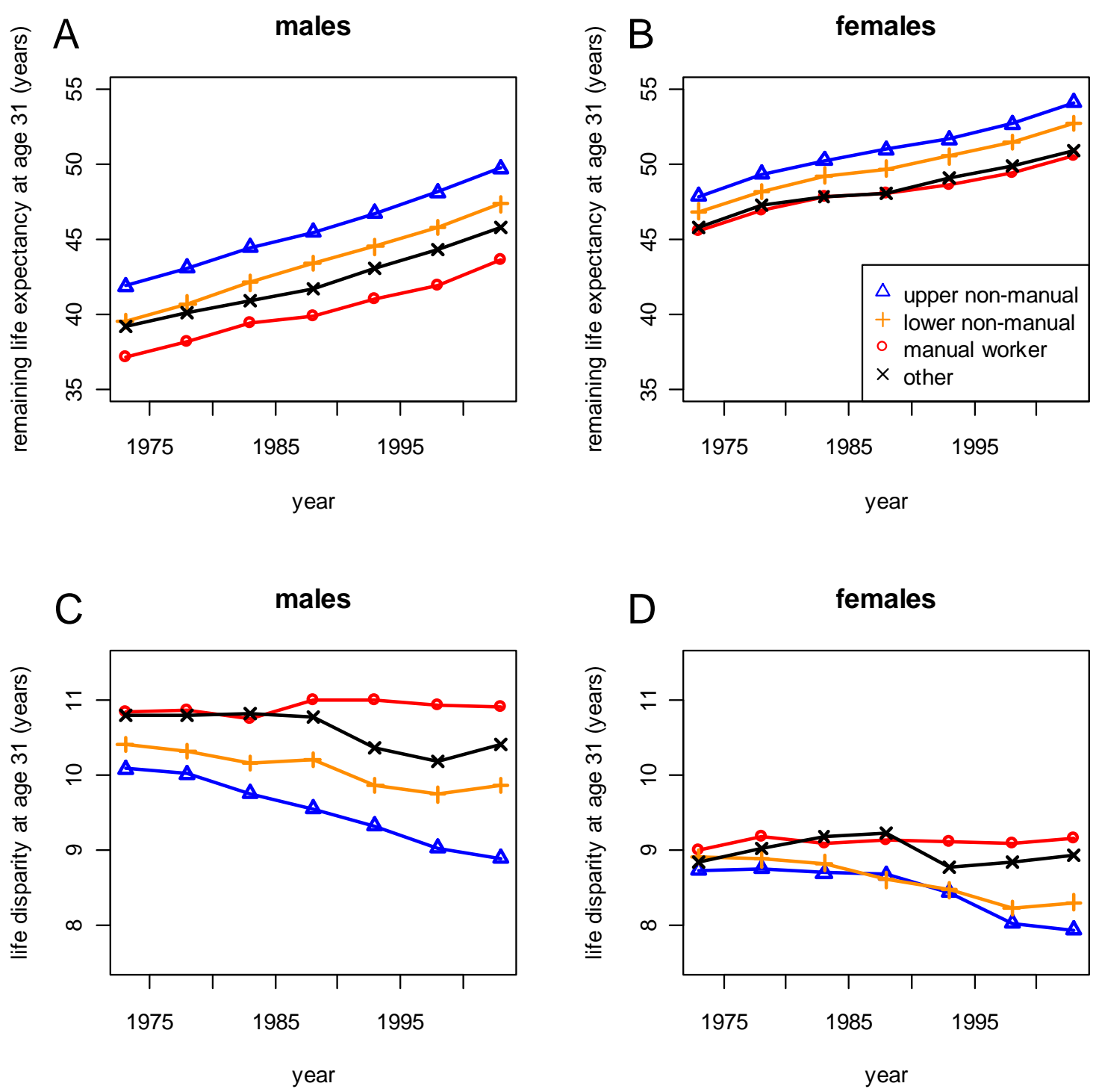

Figure 3: Trends in remaining life expectancy (upper panels) and life disparity (lower panels) at age 31 by occupational class, Finland, 1971-2007. 
A

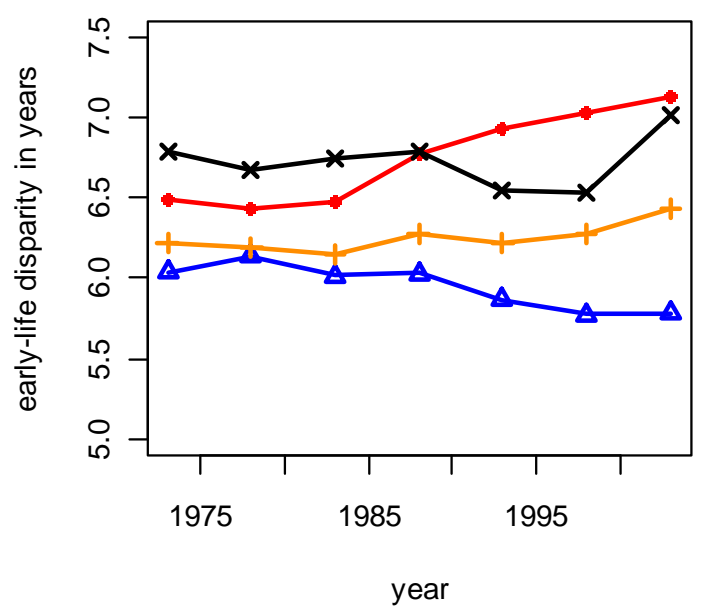

C

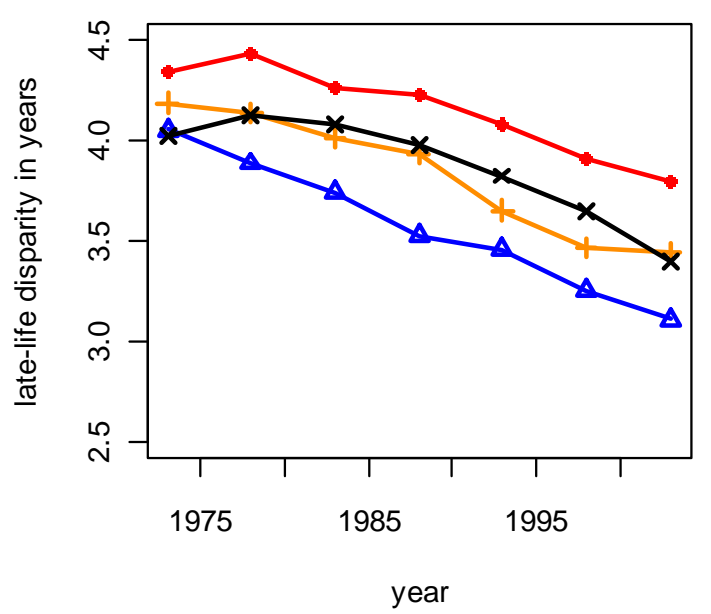

B

females

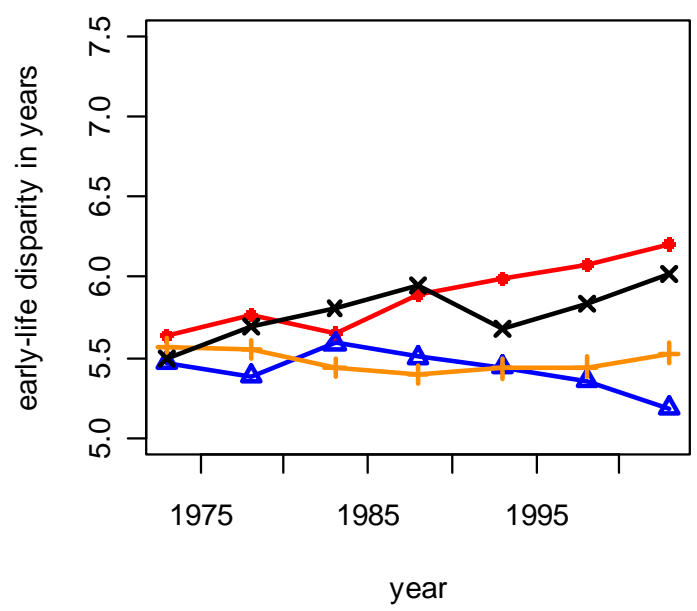

D

females

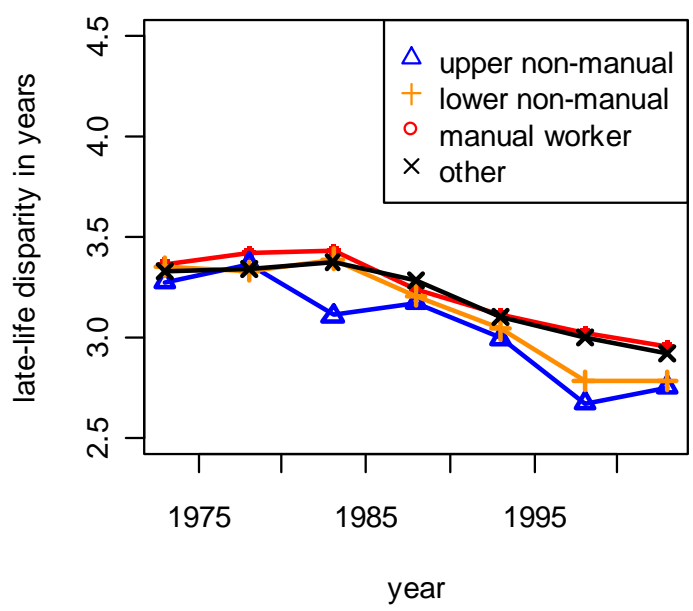

Figure 4: Trends in early-life disparity (top), and late-life disparity (bottom), at age 31 by occupational class, Finland, 1971-2007. 

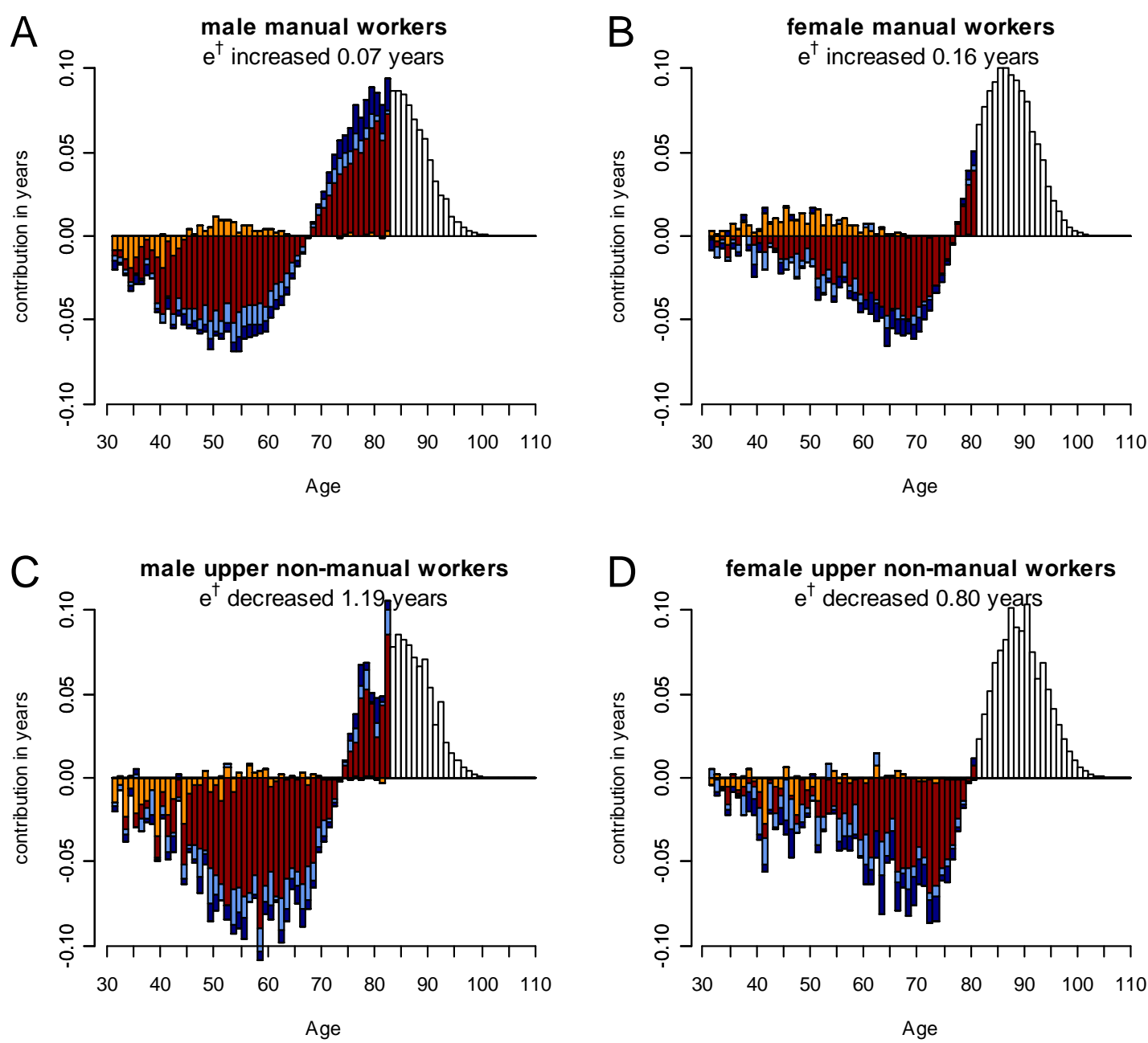

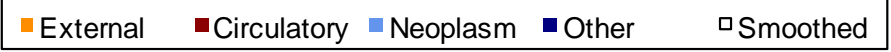

Figure 5: The change in life disparity decomposed into age and cause-specific components (in years), from $1971-75$ to $2001-07$, by occupational class. ${ }^{8}$ A positive contribution implies that the cause of death at that particular age led to an increase in life disparity over the period and vice versa. The white bars begin at ages that were smoothed in the earlier distributions - as such we do not estimate the contribution of different causes over these ages.

\footnotetext{
${ }^{8}$ The total contributions from each cause of death (up to ages where smoothing took place) to the change in lifespan variation over the time period observed were as follows:

Manual workers: external mortality -0.03 years (males), 0.23 years (females); circulatory diseases -0.45 years (males), -0.88 years (females); neoplasms -0.16 years (males), -0.16 years (females); other causes 0.02 years (males), -0.16 years (females)

Non-manual workers: external mortality -0.24 years (males), -0.13 years (females); circulatory diseases 1.24 years (males), -1.10 years (females); neoplasms -0.30 years (males), -0.37 years (females); other causes -0.14 years (males), -0.32 years (females)
} 
A

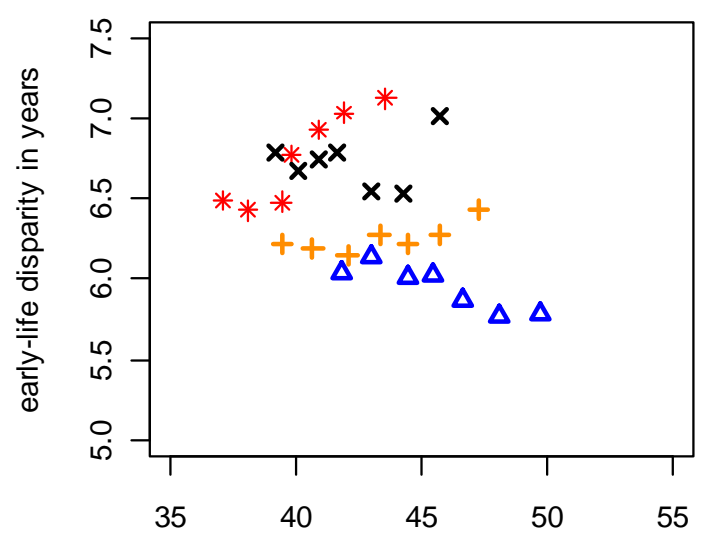

remaining life expectancy at age 31

$$
\text { C }
$$

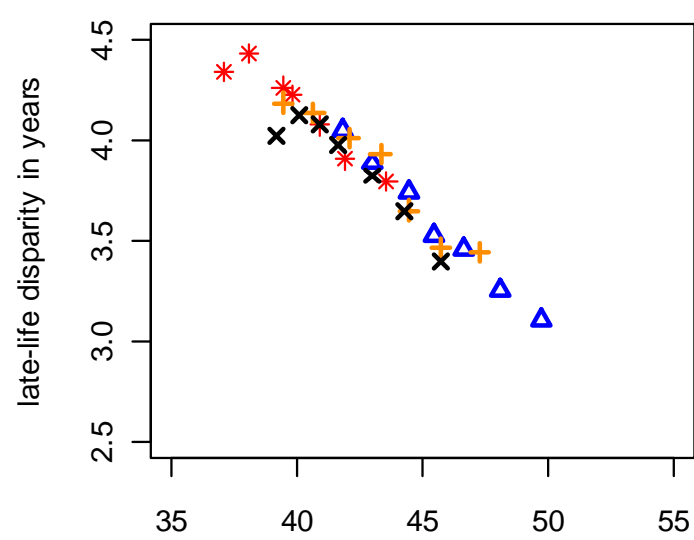

remaining life expectancy at age 31
B

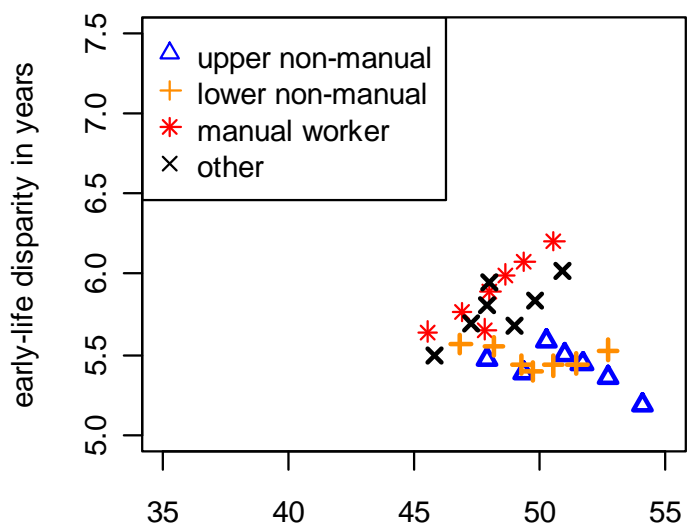

remaining life expectancy at age 31

D

females

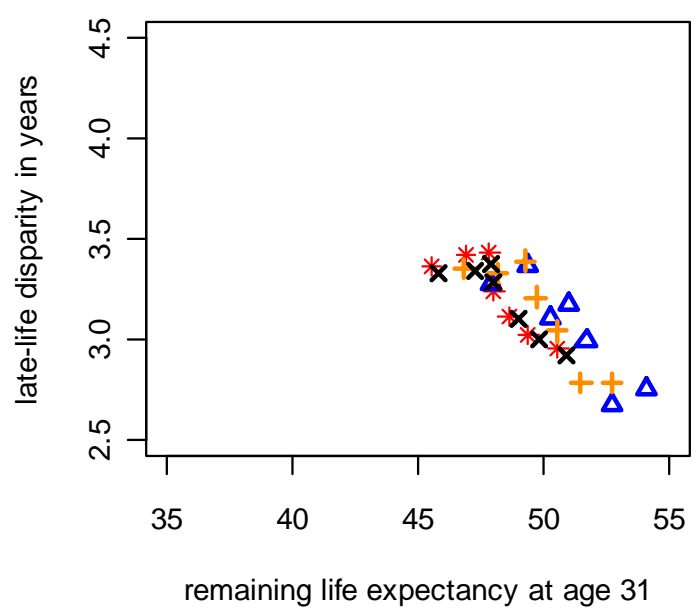

Figure 6: Trends in early and older age disparity (at age 31) at different levels of remaining life expectancy (at age 31) by occupational class, Finland, 5 year periods, 1971-2007. 

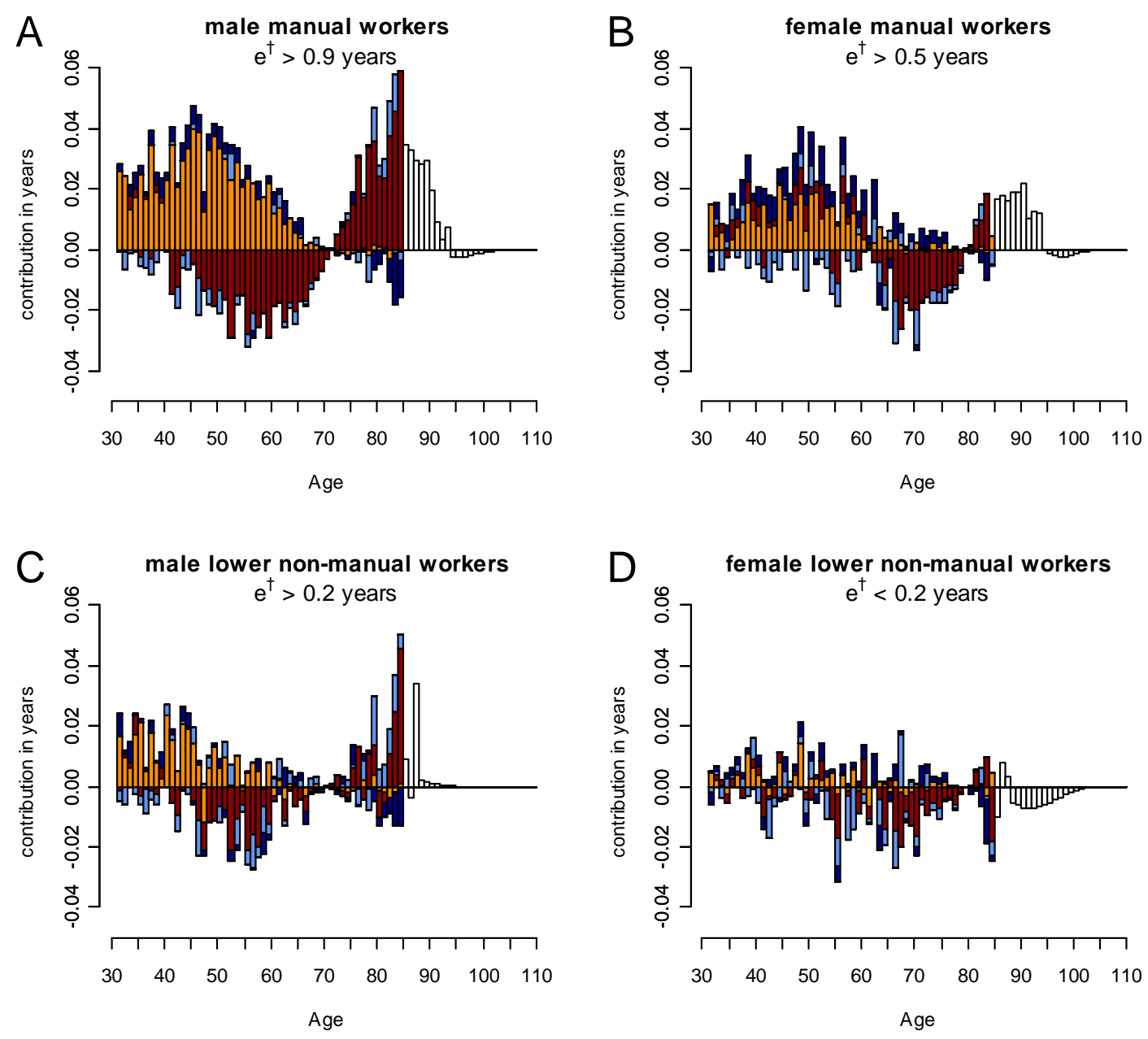

$$
\text { External } \quad \text { Circulatory } \quad \text { Neoplasm } \quad \text { Other } \quad \text { Smoothed }
$$

Figure 7: Age and cause of death decomposition of life disparity at age 31 between the upper non-manual and manual classes (top panels), and the upper non-manual and lower non-manual classes (lower panels) when remaining life expectancy at age 31 was similar (around 43 years for males, 50.5 years for females). ${ }^{9}$ A positive contribution implies that the cause of death at that particular age led to higher lifespan variation for the stated occupational class as compared to the non-manual class at this level of remaining life expectancy and vice versa.

\footnotetext{
${ }^{9}$ The total contributions from each cause of death (up to ages where smoothing took place) to the difference in lifespan variation between the stated occupational class and non-manual workers: Manual workers: external mortality 0.78 years (males), 0.35 years (females); circulatory diseases -0.12 years (males), 0.00 years (females); neoplasms -0.03 years (males), -0.10 years (females); other causes 0.08 years (males), 0.20 years (females)

Lower non-manual workers: external mortality 0.25 years (males), 0.06 years (females); circulatory diseases

-0.11 years (males), -0.16 years (females); neoplasms 0.00 years (males), -0.08 years (females); other causes -0.00 years (males), 0.02 years (females)
} 
A

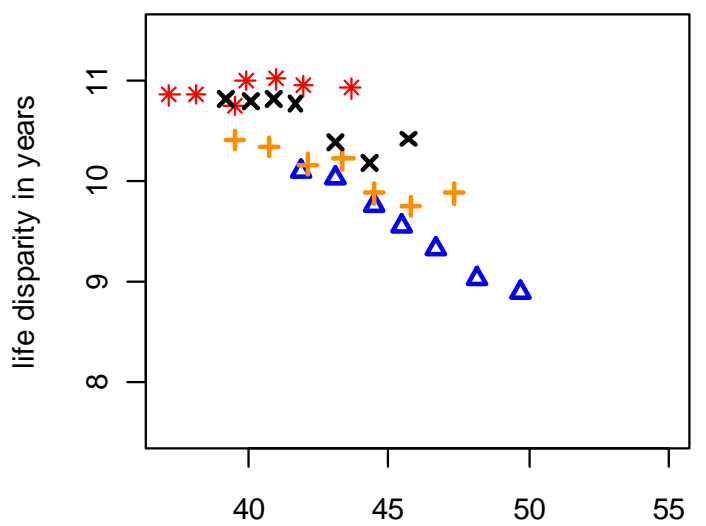

remaining life expectancy at age 31
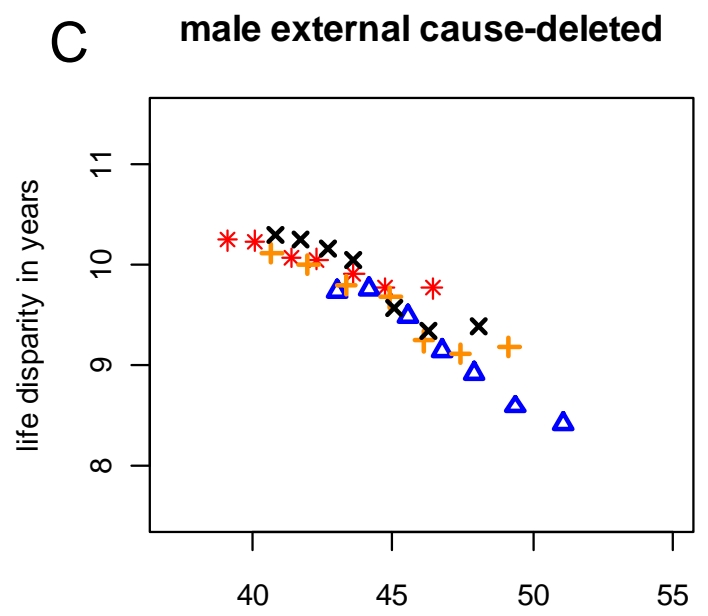

remaining life expectancy at age 31
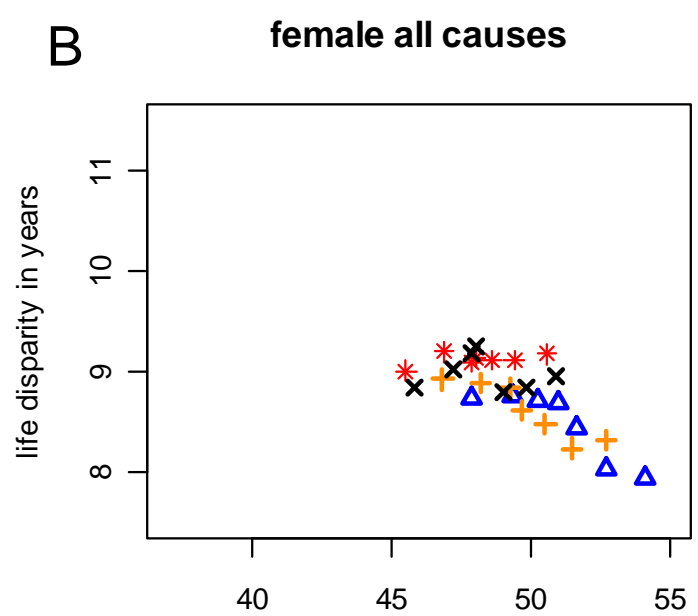

remaining life expectancy at age 31
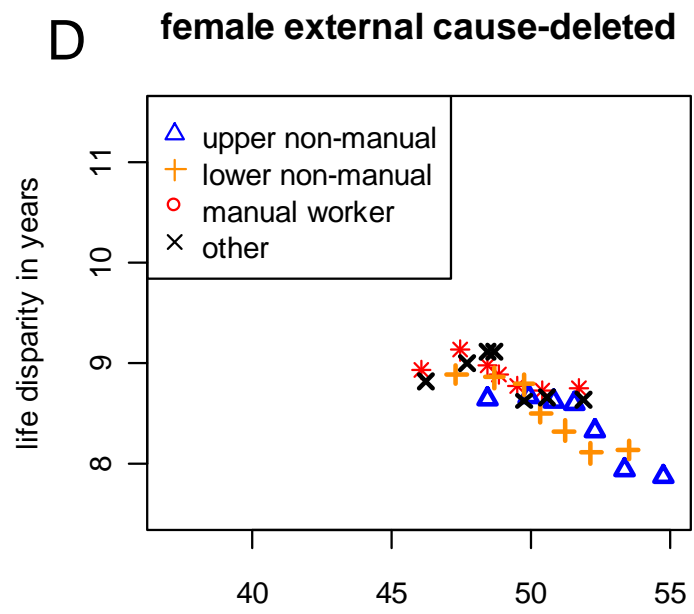

remaining life expectancy at age 31

Figure 8: Life disparity at age 31 at different levels of remaining life expectancy at age 31, comparing all-cause mortality (top panels) to external cause-deleted mortality (bottom panels). Each point refers to a time point. 